\title{
Nichts Neues an der Spitze der Großunternehmen!? Die deutsche Wirtschaftselite zwischen 1970 und 2020
}

\author{
Michael Hartmann
}

Angenommen: 1. März 2021 / Online publiziert: 24. März 2021

(C) Der/die Autor(en) 2021

Zusammenfassung Um die Jahrtausendwende wurde vielfach das Ende des spezifisch deutschen Modells der Managerrekrutierung und eine Angleichung an die angelsächsischen Verhältnisse verkündet. Der vorliegende Aufsatz überprüft diese These für die Vorstandsvorsitzenden der 100 größten deutschen Unternehmen anhand der zentralen Merkmale Ausbildung, Karrieretypus, Internationalität und soziale Herkunft zu fünf Zeitpunkten: 1970, 1995, 2005, 2015 und 2020. Es zeigt sich, dass die Rekrutierungskriterien und -mechanismen weit stabiler sind als seinerzeit angenommen. Es dominiert nach wie vor die Hauskarriere, und die Ingenieure und Naturwissenschaftler haben ihre Position sogar ausbauen können. Auch bei der sozialen Herkunft sind keine wesentlichen Veränderungen zu verzeichnen. Das gilt ebenfalls für die Aufsichtsratsvorsitzenden: Bei ihnen wie bei den Vorstandschefs stammen den gesamten Zeitraum hindurch über vier Fünftel aus bürgerlichen oder großbürgerlichen Familien. Die einzige gravierende Veränderung liegt im völligen Bedeutungsverlust der Juristen unter den Vorstandsvorsitzenden. Der Anteil der Ausländer unter ihnen wiederum ist zwar bis 2015 deutlich auf knapp $15 \%$ angestiegen, in den letzten fünf Jahren aber wieder auf das Niveau von 2005 mit knapp $10 \%$ zurückgefallen.

Schlüsselwörter Wirtschaftselite · Karrieretypus · Bildungswege ·

Internationalität · Soziale Herkunft · Modell Deutschland

\footnotetext{
M. Hartmann $(\triangle)$

Institut für Soziologie, Technische Universität Darmstadt, Dolivostraße 15, 64293 Darmstadt, Deutschland

E-Mail: hartmann@ifs.tu-darmstadt.de
} 


\title{
Nothing new at the top!? The German economic elite between 1970 and 2020
}

\begin{abstract}
Around the turn of the millennium, it was frequently proclaimed that the specific German model of manager recruitment was coming to its end, following an alignment with Anglo-Saxon conditions. This paper reviews this thesis for the CEOs of the 100 largest German companies, based on the central characteristics of training, career type, internationality and social background at five points in time: 1970, 1995, 2005, 2015 and 2020. It turns out that the recruitment criteria and mechanisms are much more stable than was assumed at the time. In-house careers continue to dominate and engineers and scientists have even been able to expand their position. Regarding social background, nothing of importance has changed, either. This also applies to the chairmen of the supervisory boards. Among them, as among the CEOs, over four fifths come from upper middle-class or upper class families throughout the entire period. The only significant change is the complete demotion of lawyers among the CEOs. Although the proportion of foreigners among them has risen significantly to nearly $15 \%$ by 2015 , in the last five years it has fallen back to the 2005 level of hardly $10 \%$.
\end{abstract}

Keywords Business elite $\cdot$ Career type $\cdot$ Educational paths $\cdot$ Internationality Social background · Model Germany

\section{Rien de nouveau à la tête des grandes entreprises !? L'élite économique allemande entre 1970 et 2020}

Résumé Au début du millénaire, on a souvent annoncé la fin du modèle spécifiquement allemand de recrutement des dirigeants et un alignement sur les pratiques anglo-saxonnes. Cet article vérifie si cette thèse est exacte pour les présidents des directoires des 100 plus grandes entreprises allemandes sur la base des principales caractéristiques que sont la formation, le type de carrière, l'internationalité et l'origine sociale à cinq moments: en 1970, 1995, 2005, 2015 et 2020. Il apparaît que les critères et les mécanismes de recrutement sont bien plus stables qu'on le supposait alors. La carrière au sein d'une même entreprise prévaut toujours et les ingénieurs et les diplômés en sciences naturelles ont même pu renforcer leur position. En matière d'origine sociale, aucun changement essentiel n'est à noter. Cela vaut également pour les présidents des conseils de surveillance: Sur l'ensemble de la période, quatre cinquièmes d'entre eux comme des présidents de directoires sont issus de familles de la bourgeoise ou de la haute bourgeoise. Le seul changement de grande ampleur tient à la chute du nombre de juristes parmi les présidents de directoires. La proportion d'étrangers parmi eux a certes nettement augmenté jusqu'en 2015 pour s'établir à presque $15 \%$. Ces cinq dernières années, elle est cependant retombée au niveau de 2005 , soit presque $10 \%$.

Mots-clés Élite économique · Type de carrière · Parcours scolaire · Internationalité · Origine sociale · Modèle allemand 


\section{Einleitung}

Das halbe Jahrhundert zwischen 1970 und 2020 war in Deutschland zwar nicht so bewegt wie der gleiche Zeitraum davor mit der Weimarer Republik, dem Nationalsozialismus, dem 2. Weltkrieg und der Gründung der Bundesrepublik, doch es war nichtsdestotrotz von etlichen gravierenden gesellschaftlichen Veränderungen geprägt. Politisch reicht die Spanne vom ersten Regierungsjahr der sozial-liberalen Koalition unter Willy Brandt über die ,geistig-moralische Wende“ von Helmut Kohl Anfang der 1980er-Jahre, die Wiedervereinigung 1990 und die erste rot-grüne Regierung unter Gerhard Schröder mit der massiven Verschärfung sozialer Unterschiede bis hin zur langen Merkel-Ära mit dem Flüchtlingszustrom, den Erfolgen der AfD und der Corona-Pandemie am Schluss. Ökonomisch reicht die Spanne von den ausgehenden Wirtschaftswunderjahren mit sehr hohen Reallohnsteigerungen über die ersten größeren Krisen in den 1970er- und 1980er-Jahren, den Wiedervereinigungsboom im Westen bei gleichzeitiger großflächiger De-Industrialisierung im Osten in den 1990er-Jahren und die Finanzkrise 2008 bis hin zu den aktuellen Corona-Lockdowns. Gleichzeitig bildeten die beiden Jahrzehnte zwischen dem Untergang des realsozialistischen Lagers Ende der 1980er-Jahre und der Finanzkrise Ende der Nullerjahre die Hochphase der Globalisierung, die dann vor allem seit Mitte des zweiten Jahrzehnts durch eine wachsende Renationalisierung gerade in der britischen und der US-Regierungspolitik zunehmend unter Druck geraten ist.

All diese gesellschaftlichen Veränderungen müssten eigentlich auch Konsequenzen für die Rekrutierung und die Lebenswege der Elitemitglieder in Deutschland haben; doch so plausibel Wolfgang Schluchters Feststellung, dass die „Beschaffenheit der Führungsgruppen (...) gleichsam auf die Beschaffenheit der Gesellschaft" zurückweise (Schluchter 1963, S. 249), auch ist, so harrt sie doch einer empirischen Überprüfung. In welchem Maße dies tatsächlich der Fall ist, soll eine Analyse der deutschen Wirtschaftselite im Zeitraum der letzten 50 Jahre zeigen; denn diese war unter den zentralen Eliten - abgesehen von der politischen - wohl dem größten Veränderungsdruck ausgesetzt.

\section{Die Globalisierung, das Ende der Deutschland AG und die deutsche Wirtschaftselite}

In der sozialwissenschaftlichen Debatte hierzulande spielte die Wirtschaftselite einzig in den Jahren um die Jahrtausendwende, also in der Ära der rot-grünen Regierung, eine gewichtigere Rolle. Damals erfasste der heftige Streit über die bereits erfolgten und die noch anstehenden Veränderungen des deutschen Wirtschaftsund Gesellschaftsmodells, sprich die Zukunftsfähigkeit des sogenannten „Modell Deutschland“, auch die Soziologie: Die Globalisierung habe seit Beginn der 1990erJahre zu einer tief greifenden Veränderung des deutschen Systems der Unternehmensführung und -kontrolle in Richtung des angelsächsischen Modells geführt, so eine damals in zahlreichen Veröffentlichungen (vor allem von Soziologen und Soziologinnen aus dem Umfeld des Max-Planck-Instituts für Gesellschaftsforschung 
in Köln) aufgestellte Behauptung. ${ }^{1}$ In der Regel wird diese Entwicklung als „Auflösung“ oder „Abwicklung der Deutschland AG“ charakterisiert. Wolfgang Streeck und Martin Höpner als die wohl wichtigsten Vertreter dieser Position führen in ihrer Zusammenfassung des Forschungsstandes den umfassenden Wandel der Managerrekrutierung dabei als eine entscheidende Ursache für diesen (ihrer Ansicht nach schon Anfang des Jahrtausends weit fortgeschrittenen und unumkehrbaren) Prozess an (Streeck und Höpner 2003, S. 24, 32f.).

Streeck und Höpner sehen in den „Besonderheiten des Führungspersonals“ einen der Eckpfeiler der alten Deutschland AG. Die deutschen Manager ${ }^{2}$ hätten sich im Unterschied $\mathrm{zu}$ ihren britischen oder US-Kollegen über viele Jahrzehnte hinweg durch ,technische statt finanzwissenschaftliche[] Expertise sowie lange Unternehmenszugehörigkeiten und Amtszeiten“ ausgezeichnet (ebd., S. 32). Mit dieser Argumentation knüpfen Streeck und Höpner an eine Reihe von vor allem deutschen wie britischen Untersuchungen an, die hinsichtlich der Unterschiede in Ausbildung und Rekrutierungsmustern zu den gleichen oder zumindest ähnlichen Ergebnissen gelangen. ${ }^{3}$ Alle diese Autorinnen und Autoren verweisen immer wieder darauf, dass die von ihnen konstatierte Vorherrschaft der Ingenieure und Naturwissenschaftler im Topmanagement deutscher Großunternehmen ein Managementhandeln begünstige, wenn nicht sogar bedinge, das der Verfolgung produktions- und technikbezogener Ziele den Vorrang gegenüber einseitig gewinnorientierten Zielen einräume. Gleichzeitig unterstütze die überwiegend interne Rekrutierung des Managements bis in die Vorstände hinein eine auf Dauerhaftigkeit und Produktqualität statt auf das Erreichen relativ kurzfristiger Gewinnmarken angelegte Unternehmenspolitik. Beides bilde zudem einen wesentlichen Grundstein für den (für die Deutschland AG charakteristischen) weitreichenden Konsens zwischen Management und Belegschaft. Die technisch-naturwissenschaftliche Ausbildung, zumal wenn sie mit einer vorher durchlaufenen Lehre einhergehe, verschaffe Facharbeitern und Managern eine gemeinsame fachliche Basis, und die langen Unternehmenszugehörigkeiten sorgten für Interessenidentität im Sinne einer vorrangigen Orientierung auf langfristige Unternehmensstabilität. Demgegenüber führten im angelsächsischen Raum die eindeutige Dominanz finanzwirtschaftlich orientierter Wirtschaftswissenschaftler in den Topetagen und die aufgrund vorwiegend externer Rekrutierung viel kürzeren Amtszeiten der Manager zu einer Politik, die kurzfristig erzielbare Gewinne in das Zentrum des Managementhandelns stelle (Gergs und Schmidt 2002, S. 557 f.; Höpner 2004, S. 265 f.).

\footnotetext{
1 S. u. a. Beyer 1998, 1999, 2006; Hassel et al. 2000; Höpner 2003, 2004; Höpner und Jackson 2001, 2002; Streeck 2006; Streeck und Höpner 2003.

2 Der Begriff Manager umfasst hier wie auch in den meisten anderen Veröffentlichungen dieser Debatte einen weit größeren Kreis als nur die Wirtschaftselite. Zählen zur letzteren in allen großen deutschen Elitestudien äußerstenfalls die Vorstands-, stellvertretenden Vorstands- und Aufsichtsratsvorsitzenden der 250 bis 300 größten Unternehmen und die restlichen Vorstandsmitglieder nur bei den Top-14, insgesamt also höchstens gut 900 Personen (Hartmann 2013, S. 30, 228), so geht es bei den Managern um sämtliche Vorstandsmitglieder von Unternehmen, teilweise bis hin zu mittelständischen Firmen.

3 S. u. a. Byrkjeflot 2000; Gergs und Schmidt 2002; Lane 1989; Locke 1989; Sorge 1999; Stewart et al. 1994.
} 
Die veränderten Rahmenbedingungen auf den internationalen Märkten, vor allem auf den Finanzmärkten, hätten nun, so die Argumentation, seit den 1990er-Jahren zu einer Angleichung der deutschen Managementrekrutierung an die Strukturen in Großbritannien und den USA geführt, und dies vor allem in zwei Punkten. Zum einen verdrängten die Wirtschaftswissenschaftler die Ingenieure und Naturwissenschaftler von der Spitze der Konzerne, zum anderen sei auch hierzulande ein unternehmensexterner Arbeitsmarkt für Manager entstanden, der zunächst die Lockerung und schließlich das Ende der langfristigen Unternehmensbindungen zur Folge habe. Dazu komme dann noch die deutliche Reduzierung personeller Verflechtungen auf der Ebene der Aufsichtsräte, vor allem durch den Rückzug von Bankvorständen aus den Aufsichtsräten von Unternehmen anderer Branchen. Hier schlage sich die Auflösung der wechselseitigen oder, wie im Falle von Banken und Industrie, auch einseitigen Kapitalbeteiligungen nieder (Beyer 2006, S. 188 ff.). Außerdem, und hier knüpft Beyer an die Diskussion über die Studienfächer der Vorstandsvorsitzenden an, wichen jene Unternehmen, die von einem Manager mit einem wirtschaftswissenschaftlichen Abschluss geführt würden, ,in signifikanter Weise vom Durchschnitt $\mathrm{ab}^{\circ}$, und zwar durch ein deutlich geringeres Maß an Verflechtungen (ebd., S. 194).

In der gesamten Debatte spielte die soziale Rekrutierung der Manager erstaunlicherweise überhaupt keine Rolle, obwohl sie für den als typisch deutsch charakterisierten Konsens zwischen Management und Belegschaft von nicht unerheblicher Bedeutung sein müsste. Gerade der Vergleich mit den als ausgesprochen elitär geltenden, in expliziten Eliteschulen und -hochschulen ausgebildeten britischen Topmanagern hätte die genannten Autoren und Autorinnen eigentlich dazu veranlassen müssen, diesen Aspekt zu thematisieren und näher zu beleuchten. Schließlich galt hierzulande eine Lehre, ein anschließendes Ingenieurstudium und das Absolvieren der gesamten Berufskarriere in einem einzigen Unternehmen für Mittelschicht- und vor allem für Arbeiterkinder stets als der Königsweg, wollten sie es bis ins obere Management schaffen. Sollte die These von Streeck und anderen stimmen, dass Lehre, ingenieurwissenschaftliches Studium und Hauskarriere massiv an Gewicht verlieren, müsste das dementsprechend auch spürbare Folgen für die soziale Zusammensetzung des Spitzenmanagements haben. Sie müsste deutlich exklusiver werden. Daran schließt sich eine weitere Frage an, die allerdings nicht die innere Verfasstheit der Wirtschaftselite, sondern ihre gesamtgesellschaftliche Rolle als eine der zwei mächtigsten gesellschaftlichen Teileliten betrifft. Die Deutschland AG war ja nicht nur durch die „Besonderheiten des Führungspersonals“ in der Wirtschaft gekennzeichnet, sondern auch durch eine spezifische Form der Elitenkooperation und -integration. Die Entstehung eines unternehmensexternen Arbeitsmarkts für Manager müsste in Verbindung mit der wachsenden Globalisierung auch in diesem Punkt für eindeutige Veränderungen sorgen. Spitzenpositionen müssten deutlich häufiger mit von außen geholten ausländischen Managern besetzt und die horizontale Integration der deutschen Eliten in ihrer Gesamtheit dadurch merklich geschwächt werden.

Diesen beiden Fragen nachzugehen und dabei die Annahmen von Streeck und anderen auf ihre Stichhaltigkeit hin zu überprüfen, ist das Ziel dieses Beitrags. Was das Untersuchungssample angeht, so sind als der Kern der deutschen Wirtschaftselite zum einen die Vorstandsvorsitzenden oder die Sprecher der Geschäftsführun- 
gen der 100 größten Unternehmen ausgewählt worden, zum anderen die Vorsitzenden der Aufsichtsräte oder vergleichbarer Gremien wie Gesellschafterausschüsse etc. derselben Unternehmen. Für die Vorstandschefs sind alle wesentlichen Angaben (Alter, soziale Herkunft, Bildungsabschlüsse, Studienfächer, Karrieremuster und Internationalität) $^{4}$ für fünf Zeitpunkte (1970, 1995, 2005, 2015 und 2020) ermittelt worden. Bei den Aufsichtsratsvorsitzenden ist die Recherche auf drei Zeitpunkte (1970, 1995 und 2020) und zwei Merkmale (soziale Herkunft und gleichzeitige Spitzenposition innerhalb des Bankensektors) beschränkt worden.

Die in die Untersuchung einbezogenen Unternehmen sind wie in den Vorläuferstudien (Hartmann 1997, 1999, 2007, 2009, 2016, 2018) anhand der seit über 60 Jahren jährlich veröffentlichten FAZ-Liste der „100 Größten“ für die entsprechenden Jahre bestimmt worden. Aus den dort aufgeführten Unternehmen sind dabei stets nur die deutschen Mutterkonzerne herausgefiltert worden, weil alle wesentlichen Entscheidungen der deutschen Wirtschaftselite stets in ihren Führungsgremien getroffen werden. Die Tochtergesellschaften in- wie ausländischer Konzerne sind deshalb unberücksichtigt geblieben.

Was die Verteilung auf die verschiedenen Branchen angeht, so hat sie sich im Laufe der Jahre ein Stück weit verändert. Gemäß der realen Veränderungen in der Gesamtwirtschaft haben die Industrieunternehmen etwas an Gewicht verloren. Stellten sie 1970 noch 56 der 100 Konzerne, so sind es aktuell nur noch 53. Zwischenzeitlich waren es sogar nur noch 50. Der neuerliche Anstieg für 2020 ist auf die gleichzeitig erfolgende deutliche Reduzierung des Anteils der Finanzunternehmen zurückzuführen. Machten sie in den früheren Jahren immer ein Fünftel der 100 Unternehmen aus, so ist ihre Zahl diesmal auf zehn halbiert worden. Das reflektiert in erster Linie den enormen Bedeutungsverlust der deutschen Banken im internationalen Finanzgeschäft wie auch in der deutschen Wirtschaft allgemein. Spielten bis in das erste Jahrzehnt dieses Jahrtausends mit Deutscher Bank, Dresdner Bank und Commerzbank noch drei große deutsche Privatbanken auch international eine wichtige Rolle, so gilt dies aktuell bestenfalls noch für die Deutsche Bank, aber auch bei ihr mit dramatisch gesunkenem Einfluss. Da die ehemals für die Industriepolitik hierzulande ausgesprochen wichtigen Landesbanken wie vor allem die WestLB im letzten Jahrzehnt gleichfalls massiv an Bedeutung verloren haben und der Beitrag des Finanzsektors zum Bruttoinlandsprodukt ebenfalls deutlich zurückgegangen ist, sind nur noch vier Banken im Sample vertreten. Deutlich dazu gewonnen haben seit 1970 dagegen die Dienstleistungs- und Handelskonzerne. Sie haben ihren Anteil kontinuierlich von anfangs 24 auf inzwischen 37 Unternehmen steigern können, d.h. um über $50 \%$.

\footnotetext{
4 Für 2015 fehlen Angaben zur sozialen Herkunft, weil die Untersuchung sich damals auf die Internationalität der Topmanager in verschiedenen Ländern konzentriert hat (Hartmann 2016), und ganz generell fehlt die Kategorie Geschlecht, weil aktuell nur zwei dieser Positionen von Frauen besetzt werden, bei ThyssenKrupp und bei Braun. Bei den Aufsichtsratsvorsitzen sind es mit vier (bei Henkel, Würth, Brenntag und Zalando) auch nicht viel mehr. Vier dieser sechs Frauen sind im Übrigen Mitglieder der Familien, die die Unternehmen kontrollieren. 1970 und 1995 gab es sogar überhaupt keine in einer solchen Position. Im gesamten Sample stellen Frauen also weniger als ein Prozent der Personen. Aus diesem Grund wird auch sprachlich durchgängig die männliche Form gewählt.
} 
Als Zeitpunkt für die konkrete Identifizierung des Spitzenpersonals der Unternehmen ist jeweils der September des entsprechenden Jahres gewählt worden (weil die FAZ-Liste seit längerem zwar im Juli erscheint, ursprünglich aber immer im September erschien, so auch für das Jahr 1970). Insgesamt sind die Daten für 498 Vorstandsvorsitzende ${ }^{5}$ und 277 Aufsichtsratsvorsitzende ${ }^{6}$ erhoben worden, insgesamt also 775 Personen. Die Ausschöpfungsquoten liegen für die meisten Merkmale bei $100 \%$ oder knapp darunter. So waren etwa 1970 für zwei Personen die Bildungsabschlüsse oder 2020 für eine Person das genaue Geburtsjahr nicht herauszubekommen. Einzig bei der sozialen Herkunft fallen die Quoten deutlich niedriger aus. So sind es bei den Vorstandsvorsitzenden ,nur“ zwischen rund 71 (für 2005 und 2020) und rund $87 \%$ (für 1970 und 1995). Bei den Aufsichtsratsvorsitzenden liegen die Werte mit knapp 74 bis gut $76 \% 7$ hingegen nahe beieinander, fallen die Differenzen zwischen den Jahrgängen kaum ins Gewicht. Alles in allem sind das Ausschöpfungsquoten weit oberhalb des in Studien über die Wirtschaftselite üblichen Niveaus.

\section{Die Bildungs- und Karrierewege der Vorstandsvorsitzenden}

Die Bildungs-und Karrierewege der Vorstandsvorsitzenden zeigen in ihren einzelnen Merkmalen sowohl deutliche Veränderungen als auch erstaunliche Stabilität. Was zunächst überrascht, ist die relativ gleichbleibende Alterszusammensetzung. Entgegen einer weitverbreiteten Meinung haben zu allen Zeitpunkten um die zwei Drittel der Vorstandschefs mindestens das fünfundfünfzigste Lebensjahr abgeschlossen, 1970 etwas mehr, seit 1995 etwas weniger. Nur etwa ein Fünftel dagegen ist 50 oder jünger, auch hier 1970 erstaunlicherweise mehr als in den anderen Jahren. ${ }^{8}$ Unter 40 ist kaum jemand und wenn, dann so gut wie immer ungewöhnlich früh an die Spitze beförderte Söhne der Familien, denen die Unternehmen (oft schon seit Generationen) gehören. Was sich zwischen 1970 und 1995 verändert hat - seither gibt es nur noch geringe Schwankungen -, ist allerdings der Prozentsatz der über 60-jährigen. Brachten sie es 1970 noch auf gut ein Drittel, so ist es seit 1995 nur noch ein knappes Viertel. Hier machen sich die in den meisten Unternehmen mittlerweile gültigen Altersbeschränkungen für Vorstandspositionen bemerkbar. Von der oft beschworenen Verjüngung der Führungsspitzen kann aber dennoch keine Rede sein.

Die Stabilität der Altersstruktur spricht schon auf den ersten Blick dafür, dass sich auch die Karrieremuster nicht sonderlich stark verändert haben. Bei genauerem Hinsehen bestätigt sich diese Annahme (Abb. 1). Es dominiert nach wie vor die

\footnotetext{
51970 und 1995 gab es vier bzw. drei Unternehmen ohne Vorstandsvorsitzenden oder-sprecher, 2015 und 2020 zwei bzw. drei Unternehmen mit jeweils zwei Vorstandsvorsitzenden.

61970 gab es 16, 1995 noch sieben Unternehmen ohne eine solche Position.

${ }^{7}$ Diese Werte beziehen sich auf die Personen. Bezieht man sie auf die Positionen, erhöhen sie sich aufgrund etlicher Doppel- oder gar Mehrfachmandate auf 77 bis $82 \%$.

81970 fällt der Anteil der 51- bis 55-jährigen besonders gering aus. Das dürfte vor allem mit der drastisch gesunkenen Geburtenrate während des 1. Weltkriegs und kurz danach zu erklären sein. Sie lag von 1915 bis 1919, also in den entsprechenden Altersjahrgängen, um ein knappes Drittel bis über die Hälfte unter den zuvor üblichen Werten (Petzina et al. 1978, S. 32).
} 
Abb. 1 Die Karriereverläufe der Vorstandsvorsitzenden der 100 größten deutschen Unternehmen. (Quelle: eigene Berechnungen)

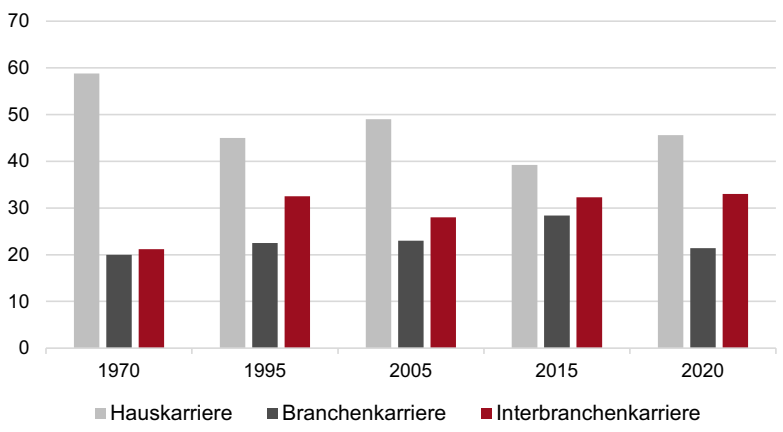

Hauskarriere, bei der der spätere Vorstandsvorsitzende spätestens vier Jahre nach Ausbildungsabschluss in das Unternehmen eingetreten ist, dem er später vorsteht, und es bis zum Erreichen dieser Position auch nicht mehr verlassen hat. ${ }^{9}$ Fast jeder zweite Vorstandsvorsitzende hat eine solche Laufbahn absolviert. Der Anteil der Hauskarrieren liegt allerdings erst seit 1995 weitgehend stabil auf diesem Niveau. Zwischen 1970 und 1995 ist er dagegen noch um ein knappes Viertel von 58,8 auf $45 \%$ gesunken.

Die naheliegende Vermutung, dass der Bedeutungsverlust der Hauskarrieren mit dem gesunkenen Anteil an über 60-jährigen Vorstandsvorsitzenden zu erklären ist, geht m.E. jedoch in die Irre. Für diesen Prozess dürfte im Wesentlichen etwas anderes verantwortlich sein, nämlich das Aufkommen der Unternehmensberatungen als wichtige erste Karrierestation. Würde man die Definition der Hauskarriere nämlich weiter fassen und alle die Vorstandschefs mit hineinnehmen, die zunächst bei einer Unternehmensberatung angefangen haben und dann nach einigen Jahren dort - oft schon beim späteren Arbeitgeber eingesetzt - in das jetzige Unternehmen gewechselt sind, dann läge der Anteil der Hauskarrieren um ungefähr zehn Prozentpunkte höher, also auf praktisch demselben Niveau wie 1970. Für diese Vermutung spricht auch, dass der Anteil der Interbranchenkarrieren, d.h. von Karrieren mit einem zwischenzeitlichen Wechsel der Beschäftigungsbranche, seit 1970 um nahezu denselben Prozentsatz zugelegt hat, von 21,2 auf $33 \%$. Hinter dieser Zunahme verbirgt sich in der Regel ein Wechsel aus der Beratungsbranche in andere Dienstleistungsunternehmen, die Industrie oder den Handel. Der Anteil der reinen Branchenkarrieren ist hingegen mit aktuell 21,4 gegenüber $20 \%$ vor 50 Jahren über ein halbes Jahrhundert fast gleichgeblieben.

Alles in allem hat sich nach den geschilderten Veränderungen zwischen 1970 und 1995 seither in den Karrieremustern (mit leichten Schwankungen) so gut wie nichts mehr getan. Die Werte für 1995 und 2020 sind fast identisch. Die Hauskarrieren machen heute 45,6 statt wie damals $45 \%$ aus, die Branchenkarrieren 21,4 statt

\footnotetext{
9 Die Definition von Hauskarriere ist hier strikter als in den meisten anderen Studien, weil sie so aussagekräftiger ist in Hinblick auf die Debatte um ein Ende des spezifisch deutschen Karrieremodells. So ist deshalb z.B. Christian Sewing, der Vorstandsvorsitzende der Deutschen Bank, nicht unter die Kategorie Hauskarrieren eingruppiert worden, obwohl er schon die Lehre bei diesem Institut gemacht hat und danach bis auf die Jahre 2005 bis 2007, als er im Vorstand der deutschen Genossenschafts-Hypothekenbank saß, immer dort tätig war. Er war es eben nicht ununterbrochen.
} 


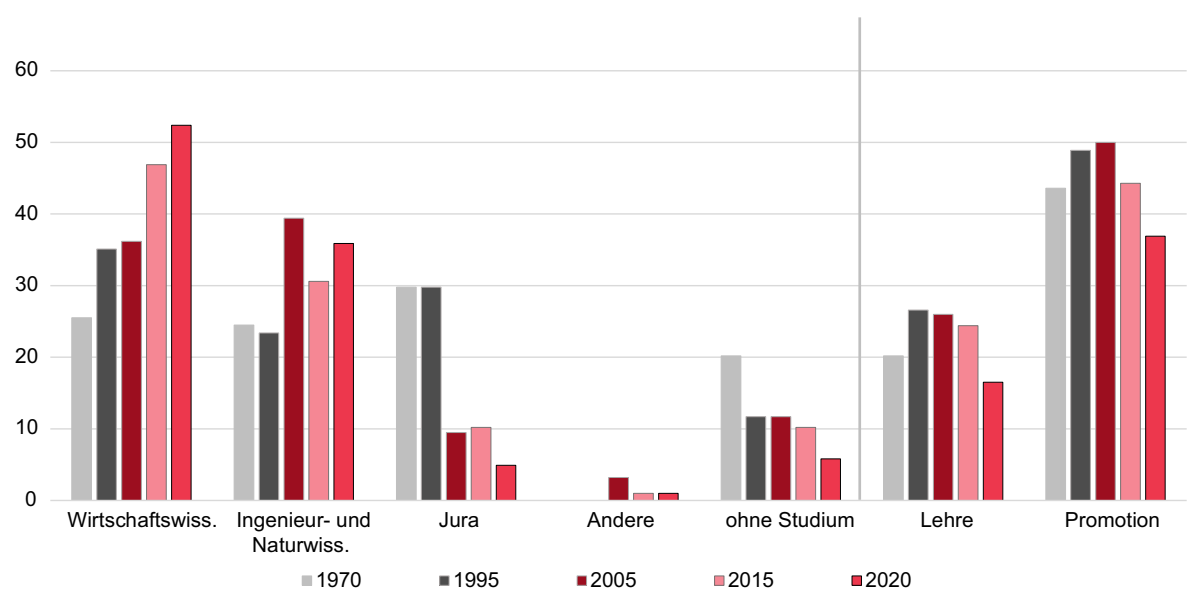

Abb. 2 Die Bildungsabschlüsse der Vorstandsvorsitzenden der 100 größten deutschen Unternehmen. (Quelle: eigene Berechnungen)

22,5\% und die Interbranchenkarrieren 33 statt 32,5\%. Diese Abweichungen sind für einen Zeitraum von einem Vierteljahrhundert minimal. Berufliche Aufstiege, bei denen jemand auch außerhalb der Wirtschaft tätig war, bilden heute genauso wie schon in den letzten Jahrzehnten eine absolute Ausnahme.

So vergleichsweise unspektakulär und einheitlich das Bild ist, das sich bei den Karriereverläufen bietet, so uneinheitlich und teilweise auch spektakulär sieht es bei den Bildungswegen aus (Abb. 2). Es gibt Merkmale mit einer relativ hohen Stabilität, solche mit allmählichen, begrenzten Veränderungen (ohne und mit eindeutiger Tendenz) und solche, die einem tiefgreifenden und grundlegenden Wandel unterworfen sind. Weitgehend unverändert über den gesamten Zeitraum von 50 Jahren zeigen sich die folgenden drei: Wer ein anderes als ein wirtschafts-, ingenieur-, natur- oder rechtswissenschaftliches Studium abgeschlossen hat, der schafft es nur in ganz seltenen Ausnahmefällen (wie etwa beim Springer-Chef Mathias Döpfner) an die Spitze eines der großen Konzerne. Dasselbe gilt für die Absolventen von privaten Business Schools, die sich selbst als Elitehochschulen und Kaderschmieden verstehen. Die EBS in Oestrich-Winkel und die WHU in Vallendar stellen jeweils einen Vorstandsvorsitzenden, ${ }^{10}$ d.h. nicht mehr oder sogar weniger als die ungefähr gleich alten Universitätsneugründungen in Augsburg, Bayreuth, Regensburg oder Passau. Es führen nach wie vor mit weitem Abstand die klassischen TUs und Universitäten wie Aachen, Karlsruhe, Köln oder München. Auch der MBA bleibt für die deutschen Topmanager als Abschluss bedeutungslos, zumindest solange er nicht den Diplombetriebs- oder -volkswirt als Regelabschluss an den staatlichen deutschen Universitäten ersetzt hat.

Den anderen Pol, den mit den gravierendsten Veränderungen, bilden der völlige Wegfall von Karrieren ohne Hochschulabschluss und der rasante Niedergang der

10 Dasselbe gilt auch für St. Gallen, wo vor fünf Jahren noch ganz überraschend gleich drei der deutschen Vorstandsvorsitzenden ihr Studium absolviert hatten. Das war offensichtlich eine Zufallskonstellation und kein Trendanzeichen. 
Juristen. Was den ersten Punkt betrifft, so hat es nur noch gut jeder zwanzigste Vorstandschef ohne ein abgeschlossenes Studium bis ganz nach oben geschafft. ${ }^{11}$ Vor 50 Jahren traf das noch auf jeden fünften $\mathrm{zu}^{12}$ und selbst vor fünf Jahren galt es noch für jeden zehnten. Hier macht sich die Bildungsexpansion unmissverständlich bemerkbar, die den Verzicht auf ein Studium von einer durchaus diskutablen Möglichkeit zu einem schwer erklärbaren Ausnahmefall degradierte. Ein Topmanager hat mir das in einem Interview mal kurz und knapp in dem Satz zusammengefasst: „In unseren Jahrgängen konnte man sich dem Studium ja quasi nur durch Selbstmord entziehen." Diese Aussage macht deutlich, warum es unter den jüngeren Vorstandsvorsitzenden so gut wie niemand mehr gibt, der nicht studiert hat.

Noch deutlich stärker als das Ende der Nichtstudierten an den Unternehmensspitzen fällt der Niedergang, fast möchte man sagen Untergang der Juristen aus. Sie stellten über lange Jahrzehnte die führende Akademikergruppe in der Gesellschaft insgesamt - man denke hier nur an das Juristenmonopol im öffentlichen Dienst - wie auch in der Wirtschaft. Bis Mitte der 1990er-Jahre hatte fast jeder dritte Vorstandsvorsitzende Jura studiert. In den Vorständen der großen Banken und Versicherungen dominierten sie sogar ganz eindeutig. Das änderte sich radikal binnen nur eines Jahrzehnts, und dieser Trend setzt sich bis heute ungebremst fort. Hatte sich ihr Anteil an den Vorstandschefs bis 2005 schon gedrittelt, so ist er mittlerweile auf unter 5\% abgerutscht. Selbst im Finanzsektor stellen Juristen inzwischen eine Rarität dar. Unter den Vorstandsvorsitzenden der zehn größten Finanzinstitute findet man gerade noch einen Juristen, und in den kompletten Vorständen der einzelnen Banken und Versicherungen sieht es nicht viel anders aus. Die Wirtschaftswissenschaftler haben sie vollkommen verdrängt, ein Prozess, der in seinen Anfängen schon in der zweiten Hälfte der 1980er-Jahre zu erkennen war und bei genauer Analyse schon damals unumkehrbar erschien (Hartmann 1990).

Der unaufhaltsame Aufstieg der Wirtschaftswissenschaftler - die ihren Anteil seit 1970 von 25,5 auf 52,4\% haben verdoppeln können - stellt eine jener Veränderungen dar, die zwar nicht rasant, dafür aber kontinuierlich verlaufen sind und eine klare Richtung aufweisen. Ähnlich, wenn auch in deutlich geringerer Ausprägung, sieht es bei den Promotionen aus. Ihre Bedeutung ist langsam, aber sicher gesunken. War bis Mitte der Nullerjahre sogar noch ein leichter Anstieg zu verzeichnen - auch das im Wesentlichen wohl eine Folge der Bildungsexpansion und der mit ihr einhergehenden Entwertung des normalen Hochschulabschlusses sowie des Versuchs, dieser Entwertung durch den Erwerb eines höher angesiedelten Bildungstitels entgegenzuwirken -, so geht es seither bergab. Aktuell hat mit knapp 37\% nur noch gut jeder dritte Vorstandsvorsitzende promoviert statt wie vor 15 Jahren jeder zweite. Der Doktortitel verliert eindeutig an Bedeutung, allerdings nur relativ langsam und nicht begleitet von einer Zunahme bei alternativen Abschlüssen wie dem MBA.

\footnotetext{
11 Studium bedeutet dabei fast immer Universitätsstudium. Absolventen von Fachhochschulen oder dualen Hochschulen sind mit einem halben Dutzend an der Zahl so selten wie auch in den letzten zwei Jahrzehnten.

${ }^{12}$ Die Differenz in diesem Punkt und beim Anteil der Lehrabschlüsse gegenüber älteren Veröffentlichungen resultiert aus Nachrecherchen für die Jahre 1970 und 1995. Diese erschienen mir notwendig, weil die Recherchemöglichkeiten damals (noch weitgehend ohne Internet-Unterstützung) deutlich schlechter waren als heute.
} 
Der Aufstieg der Wirtschaftswissenschaftler erfolgt, anders als viele Beobachter erwartet haben, jedoch nicht auf Kosten der Ingenieure und Naturwissenschaftler, sondern ausschließlich auf Kosten von Juristen und Nichtstudierten. Die Ingenieure und Naturwissenschaftler ${ }^{13}$ haben ihre Stellung über die Jahrzehnte hinweg dagegen behaupten und sogar ausbauen können. Sie haben ebenfalls vom Niedergang der Juristen profitiert. Verglichen mit dem Zeitraum zwischen 1970 und 1995, als noch fast ein Drittel der Vorstandsvorsitzenden Rechtswissenschaften studiert hatte, ist ihr Anteil von einem Viertel auf über ein Drittel gestiegen. Die Entwicklung ist zwar nicht ganz gradlinig - mit Schwankungen zwischen gut 30 und knapp $40 \%$ in den letzten 15 Jahren -, in der Tendenz jedoch eindeutig. ${ }^{14}$

Nicht ganz so eindeutig hat sich die Rolle der Lehre verändert. Hatte sie zunächst sogar noch an Bedeutung gewonnen, mit einem Zuwachs um ein Viertel seit 1970 und Werten um gut $25 \%$ zwischen 1995 und 2015, so gab es in den letzten fünf Jahren einen Einbruch um fast ein Drittel auf aktuell nur noch gut $16 \%$. Es spricht viel dafür, dass es sich dabei um eine Trendwende handelt. War die Lehre 1970 noch fast durchweg eine Alternative zum Studium - nur vier Vorstandsvorsitzende mit Studium hatten zuvor eine Lehre absolviert $-{ }^{15}$ so stellte sie über zwei Jahrzehnte zumeist eine Ergänzung zum Studium dar, die immerhin jeder vierte wählte. Die Lehre war dabei fast immer eine kaufmännische. Gewerbliche Lehren bildeten auch unter Industriemanagern eine seltene Ausnahme. Gab es schon 1995 nur ganze drei Personen, die eine solche absolviert hatten, nämlich Jürgen Schrempp bei Daimler, Peter Schumacher bei HeidelbergCement und Heinz Dürr bei der Deutschen Bahn, so gibt es heute nur noch einen einzigen, Stefan Klebert bei GEA. Mindestens ebenso häufig, zumeist sogar häufiger hatten Industriemanager erstaunlicherweise eine Banklehre abgeschlossen. Aber auch das ist heute nur noch selten anzutreffen, wie etwa bei Hubertus von Baumbach (Boehringer Ingelheim) oder Alexander Knauf (Knauf Gruppe). Die Banklehre erfreut sich nur bei Industriellendynastien immer noch einer gewissen Beliebtheit. Generell aber sorgt der höhere Zeitdruck in der Ausbildungsphase dafür, dass alle nicht unbedingt notwendigen Schritte eingespart werden, wie die Lehre, aber zum Teil auch die Promotion. Da die Manager heutzutage schneller als früher in Positionen des General Managements kommen (etwa als Geschäftsführer einer Tochtergesellschaft), spielt das Alter eine größere Rolle. Man

\footnotetext{
13 Die wenigen Mediziner unter den Vorstandschefs sind den Naturwissenschaftlern zugeordnet worden, weil sie mit diesen weit mehr verbindet als mit den Geistes- und Sozialwissenschaften und ähnlichen Fächern, die unter ,andere“ eingruppiert worden sind.

14 Zählte man die Wirtschaftsingenieure zu den Ingenieuren statt zu den Wirtschaftswissenschaftlern, würde der Abstand zu letzteren sogar auf wenige Prozentpunkte zusammenschrumpfen. Dass sie hier wie auch in den früheren Studien den Wirtschaftswissenschaftlern zugeordnet worden sind, obwohl das Studium zu gleichen Teilen aus ingenieur- und wirtschaftswissenschaftlichen Elementen besteht, hat vor allem zwei Gründe. Der erste, weniger wichtige Grund sind die eigenen Erfahrungen in der Ausbildung von Wirtschaftsingenieuren an der TU Darmstadt. Der zweite sind die Werdegänge der fünf Wirtschaftsingenieure unter den Vorstandschefs. Sie haben entweder, wie Jörg Stratmann von Mahle, anschließend in Wirtschaftswissenschaften promoviert oder aber, wie die übrigen, nie als Ingenieure gearbeitet. Typisch dafür ist Gisbert Rühl von Klöckner, der nach Studienabschluss erst zu Roland Berger ging, dann als Investmentbanker und kaufmännischer Leiter fungierte, um schließlich hohe Managementpositionen bei Rüttgers und Klöckner zu besetzen.

15 Ebenfalls vier Vorstandsvorsitzende hatten nur das Abitur ohne ein anschließendes Studium gemacht.
} 
darf nicht zu viel Zeit in der Ausbildungsphase verlieren, um in der Konkurrenz um die ersten Managementjobs nicht das Nachsehen zu haben.

\section{Die Internationalität der Vorstandsvorsitzenden}

Obwohl die Hauskarriere weiter dominant bleibt und ihr Gewicht heute genauso hoch ist wie vor 25 Jahren, externe Rekrutierungen in diesem Zeitraum also nicht häufiger geworden sind, hat die Internationalität an der Spitze der Unternehmen gleichzeitig deutlich zugenommen. Das gilt sowohl hinsichtlich der Anzahl von Ausländern in der Position des Vorstandsvorsitzenden als auch bezüglich der Auslandserfahrungen ihrer deutschen Kollegen (Abb. 3). Um mit dem ersten Punkt anzufangen: Bis Mitte der 1990er-Jahre stellten Ausländer eine seltene Ausnahme unter den Topmanagern der deutschen Großkonzerne dar. Maximal ein bis zwei der 100 größten Unternehmen wurden von Personen ohne deutsche Staatsbürgerschaft geleitet. Diese kamen zudem ausnahmslos aus dem deutschen Sprachraum. Das hat sich seit Beginn dieses Jahrtausends gravierend geändert. 2005 gab es schon neun Ausländer unter den Vorstandschefs, 2015 sogar $15 .{ }^{16}$ Mehrheitlich stammten sie zwar weiterhin aus den benachbarten und auch kulturell ähnlichen Ländern Dänemark, Niederlande, Österreich und Schweiz. Eine starke und wachsende Minderheit kam aber auch aus einem (etwas) entfernteren Kultur- und Sprachraum, zumeist aus den USA, Großbritannien oder Frankreich. 2015 traf das schon auf sechs der 15 ausländischen Vorstandschefs zu. In den letzten fünf Jahren hat sich dieser eindeutige Trend allerdings nicht fortgesetzt, sondern überraschenderweise sogar umgekehrt. 2020 stehen nur noch zehn Ausländer an der Spitze eines der Großkonzerne, fünf aus den vier genannten, kulturell eher verwandten, fünf aus anderen Ländern. Der Ausländeranteil ist damit fast wieder auf das Niveau von 2005 zurückgefallen. Die allein in den letzten vier Jahren bei den 30 DAX-Konzernen erfolgten (freiwilligen oder erzwungenen) Rücktritte von gleich sechs ausländischen Vorstandschefs zugunsten eines deutschen Nachfolgers hatten so etwas schon angedeutet. Die Liste reicht von John Cryan bei der Deutschen Bank über Marijn Dekkers bei Bayer, Hans van Bylen bei Henkel und Peter Terium bei RWE bis hin zu Bill McDermott und seiner Nachfolgerin Jennifer Morgan bei SAP. Bei den Aufsichtsratsvorsitzenden hat sich im Übrigen im gesamten Zeitraum kaum etwas geändert. Ausländer stellen nach wie vor eine seltene Ausnahme dar, wenn auch mit ganz leicht steigender Tendenz. 1995 war André Leysen bei Hapag Lloyd eine dieser Ausnahmen, 2020 sind es Hans Dieter Pötsch bei VW oder Christina Stenbeck bei Zalando.

Was die Auslandserfahrungen der deutschen Vorstandsvorsitzenden angeht, ${ }^{17}$ so ist die Entwicklung der letzten Jahrzehnte dagegen auf den ersten Blick ungebremst weiter vorangeschritten. Hatte sich der Anteil auslandserfahrener Vorstandschefs

16 Die um eine Person geringere Zahl in Hartmann 2016, S. 74 resultiert daraus, dass für dieses Buch Neubesetzungen bis März 2016 Berücksichtigung gefunden haben.

17 Als Auslandserfahrung wird gewertet, wenn jemand einmal mindestens sechs Monate am Stück im Ausland gelebt hat, entweder während der beruflichen Laufbahn, wie das zumeist der Fall ist, oder aber während des Studiums. 
Abb. 3 Die Internationalität der Vorstandsvorsitzenden der 100 größten deutschen Unternehmen. (Quelle: eigene Berechnungen)

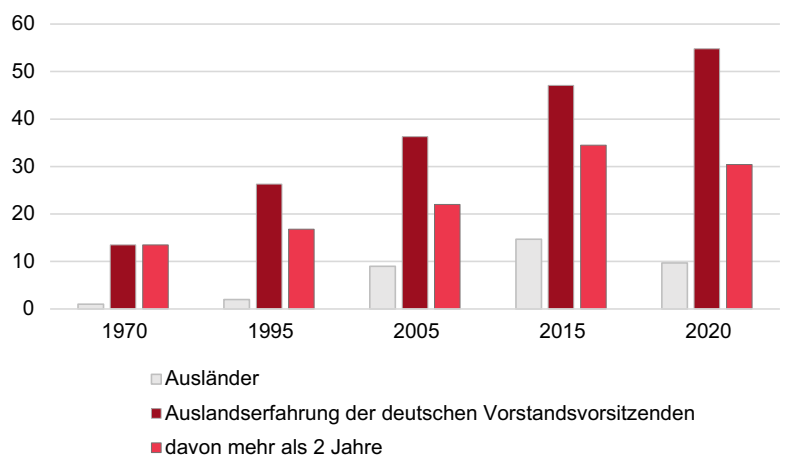

schon zwischen 1970 und 1995 von gut 13 auf gut $26 \%$ verdoppelt, so ist er seither im Schnitt pro Jahr um gut einen weiteren Prozentpunkt angestiegen, auf etwas über $36 \%$ 2005, etwas über $47 \% 2015$ und fast $55 \%$ 2020. Die extrem starke Exportorientierung der deutschen Großkonzerne und ihre weltweit massiv zugenommenen Aktivitäten dürften sich hier niederschlagen. Dennoch täuscht dieser erste Eindruck ein wenig. Gewachsen ist in den letzten Jahren nämlich nur noch die Zahl derjenigen, die maximal zwei Jahre im Ausland gelebt haben. Lag ihr Anteil an den auslandserfahrenen Vorstandschefs 2015 noch bei einem guten Viertel, so ist es aktuell schon fast die Hälfte. Demgegenüber hat sich die Zahl derjenigen unter ihnen, die mindestens zwei Jahre im Ausland gelebt haben, um über ein Zehntel reduziert. Statt gut $34 \%$ haben nur noch gut $30 \%$ zwei Jahre ihres Lebens oder mehr im Ausland verbracht - wie beim Anteil der Ausländer unter den Konzernchefs ist auch hier erstmals seit 1970 ein Rückgang in einer bis dato kontinuierlichen Aufwärtsbewegung zu beobachten. Damit liegen die deutschen Vorstandsvorsitzenden im internationalen Vergleich zwar immer noch in der Spitzengruppe (Hartmann 2016, S. 74), eine Trendwende ist aber nicht ganz von der Hand zu weisen. Bei den jüngeren deutschen Managern scheint sich in den letzten ein bis zwei Jahrzehnten eine Haltung zu verfestigen, die man wie folgt charakterisieren kann: Man muss zwar, als quasi unverzichtbarer Bestandteil einer Karriere, im Ausland tätig gewesen sein, sollte dort aber nicht zu lange bleiben; denn letztlich werden die wesentlichen Entscheidungen über die Besetzung von Spitzenpositionen immer in der Konzernzentrale getroffen. Je länger und weiter man von dort weg ist, umso größer ist das Risiko, aus dem Blickfeld der Entscheider zu geraten. Die Konsequenz ist klar: Ins Ausland gehen, ja, aber besser nur für eine relativ kurze Zeitspanne.

\section{Die soziale Rekrutierung der Wirtschaftselite}

Nimmt man das gesamte halbe Jahrhundert seit 1970 in den Blick, so weist keines der wesentlichen Merkmale eine so hohe Stabilität auf wie die soziale Rekrutierung (Abb. 4). Zu allen Zeitpunkten stammten bzw. stammen mehr als vier Fünftel der Vorstandsvorsitzenden aus Familien des Bürger- oder des Großbürgertums, die in 
60

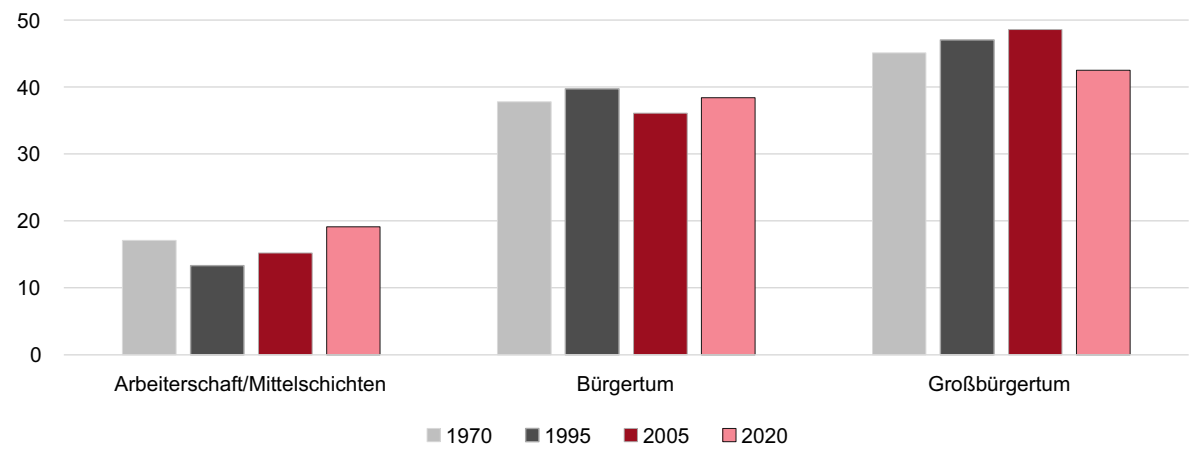

Abb. 4 Die soziale Herkunft der Vorstandsvorsitzenden der 100 größten deutschen Unternehmen. (Quelle: eigene Berechnungen)

den Geburtsjahren der Manager ungefähr $4 \%$ der Bevölkerung ausmachten. ${ }^{18}$ Die restlichen ca. $96 \%$ der Bevölkerung brachten es bestenfalls auf gut 19, schlechtestenfalls auf nur gut $13 \%$. Schaut man allein auf das Ausgangsjahr 1970 und das Schlussjahr 2020, so beträgt der Unterschied sogar nur gerade einmal zwei Prozentpunkte, etwas mehr als 19 statt etwas mehr als $17 \%$.

Für die Schwankungen im Zeitverlauf ${ }^{19}$ und das etwas höhere soziale Rekrutierungsniveau in den Jahren 1995 und 2005 ist aber nicht, wie man vermuten könnte, die Anzahl derjenigen Vorstandsvorsitzenden verantwortlich, die aus den Eigentü-

\footnotetext{
18 Je nach Geburtsjahrgang bewegt sich der Anteil zwischen 3,5 und gut 4\%. Für eine genaue Definition anhand sozialstatistischer Erhebungen wie Volkszählungen oder Mikrozensus s. Hartmann 2002, S. 36 ff., sowie Hartmann 2013, S. 201 f. In den jüngeren Jahrgängen ab Mitte der 1960er-Jahre, bei denen die Klassenzugehörigkeit nicht immer so eindeutig zu bestimmen ist wie bei den älteren, sind glücklicherweise fast durchweg Personen zu finden, bei denen eine entsprechende Zuordnung immer noch recht einfach ist. Eine große Zahl, bei den unter 50-jährigen sogar die Mehrheit, stammt aus den Eignerfamilien der Unternehmen, wie Anna Maria Braun, Ernest W. Droege, Max Heinemann, Markus Miele, Alexander Knauf oder Christoph Werner. Ein weiterer Teil kommt aus Familien, die klar dem Bürgertum zuzurechnen sind, wie Matthias Zachert von Lanxess, dessen Vater das BKA geleitet hat, Christian Klein von SAP, dessen Vater ein bekannter Politiker (Bürgermeister, Landtagsabgeordneter in Baden-Württemberg und dort Vorsitzender erst des Finanz- und Wirtschaftsausschusses, dann des Innenausschusses) ist, Toralf Haag von Voith, dessen Vater Professor an der Universität Kiel war, oder Robert Gentz von Zalando, dessen Eltern beide ein Studium abgeschlossen haben (der Vater als Tierarzt, die Mutter als Agrarökonomin) und außerdem ein altes Traditionsgestüt besitzen. Eine kleine Minderheit stammt aus den Mittelschichten, wie Christian Sewing von der Deutschen Bank oder Markus Steilemann von Covestro, deren Vätern kleine Firmen gehörten, oder aus der Arbeiterschaft, wie Robert Friedmann von Würth, der damit bei den unter 55-jährigen aber der einzige mit einer derartigen familiären Herkunft ist.

19 Die deutlich veränderte Relation zwischen Bürger- und Großbürgerkindern im Vergleich der Jahre 2005 und 2020 kann zum Teil auch darauf zurückzuführen sein, dass bei einigen Personen die Zuordnung nicht eindeutig zu klären war. So hat beispielsweise der Vorstandsvorsitzende von Schaeffler, Klaus Rosenfeld, seine Kindheit und Jugend als Sohn eines Richters am Oberlandesgericht Hamm verbracht. Er ist unter Bürgerkind eingruppiert worden, weil über die konkrete Position des Vaters nichts Näheres zu ermitteln war. Sollte dieser dort aber Vorsitzender Richter gewesen oder geworden sein, müsste Rosenfeld als Großbürgerkind zählen, weil sein Vater dann zu den nicht einmal 200 Richtern in Nordrhein-Westfalen gehört hätte, die damals in den hohen Besoldungsgruppen ab R3 eingruppiert waren.
} 
mer- bzw. Haupteignerfamilien der jeweiligen Unternehmen stammen. Das zeigt ein Vergleich der Jahre 2005 und 2020 ganz deutlich. 2005 gab es mit elf die geringste Zahl solcher Personen, 2020 mit 16 die höchste. Der Anteil der Großbürgerkinder lag dagegen 2005 mit fast $49 \%$ am höchsten und 2020 mit fast $43 \%$ am niedrigsten.

Ausschlaggebend für die exklusive soziale Rekrutierung der Vorstandsvorsitzenden sind durchgängig die Auswahlmechanismen in den Großunternehmen, die Personen aus dem Bürger- und dem Großbürgertum habituell stark begünstigen (Hartmann 1996, S. 107 ff.; Hartmann 2002, S. 117 ff.; Hartmann 2018, S. 64 ff.). Im Wesentlichen geht es dabei darum, inwieweit man mit den Gepflogenheiten der Welt vertraut ist, in die man über die Topetagen der Großkonzerne eintritt. Je souveräner und selbstverständlicher man sich dort bewegt, umso größer sind die Chancen, letztlich für Spitzenpositionen ausgewählt zu werden. Für soziale Aufsteiger stellen diese Anforderungen eine in der Regel unüberwindbare Hürde dar. Bürger- und vor allem Großbürgerkinder haben es da erheblich leichter. Auch Oberstudienräte oder Richter wie die Väter des Infineon-Chefs Reinhard Ploss und des EON-Chefs Johannes Teyssen waren in den 1950er- und den 1960er-Jahren, während der Kindheit und Jugend dieser beiden Manager, noch selbstverständlich Teil der lokalen Honoratiorenkreise in Städten wie Bamberg oder Hildesheim. Ihre Söhne waren deshalb zumindest im Großen und Ganzen vertraut mit den Gepflogenheiten dieses Milieus und speziell auch mit der Ausübung von Macht als einem zentralen Element des dazu gehörenden Habitus. Noch weit mehr gilt das natürlich für Männer, die wie die Vorstandsvorsitzenden von ZF Friedrichshafen und Noweda, Wolf-Henning Scheider und Michael P. Kuck, jemanden zum Vater hatten, der selbst schon den Vorstand eines großen Unternehmens geleitet hatte.

Bei den Aufsichtsratsvorsitzenden ist die soziale Selektivität sogar noch etwas größer (Abb. 5). Aufsteiger aus den Mittelschichten oder gar der Arbeiterschaft stellten während des gesamten Zeitraums von 50 Jahren nie mehr als knapp $17 \%$ der Amtsinhaber. Das dürfte im Wesentlichen damit zu erklären sein, dass Mitglieder der Eigentümerfamilien häufiger an der Spitze der Aufsichtsräte zu finden waren und sind als an der Spitze der Vorstände. Diese Familien üben ihre Kontrolle über die Unternehmen in der Regel lieber indirekt aus als durch direkte Beteiligung an dem für das operative Geschäft zuständigen Topmanagement. Das gilt bei Christoph Mohn genauso wie bei Georg Schaeffler, bei Simone Bagel-Trah ebenso wie bei Bettina Würth. Es gibt zusätzlich auch noch Familienmitglieder wie Jürgen Heraeus oder Michael Otto, die erst den Vorstands- und danach den Aufsichtsratsvorsitz bekleiden. Das zusammengenommen ist der Hauptgrund für den mit gut 49 bis gut $57 \%$ durchgängig höher liegenden Anteil von Personen, die in großbürgerlichen Familien aufgewachsen sind. Sie stammen mehrheitlich aus den Eigentümerfamilien. Dazu kommt dann noch eine Reihe von Männern aus demselben großbürgerlichen Milieu wie etwa Karl-Ludwig Kley, der Sohn eines Siemens-Vorstandsmitglieds, Ulrich Grillo aus einer alten Industriellenfamilie des Ruhrgebiets oder Nikolaus von Bomhard aus altem bayrischen Adel.

Dass der Prozentsatz der Bürger- und Großbürgerkinder nicht noch höher ausfällt, hat in erster Linie mit den öffentlich-rechtlich oder genossenschaftlich organisierten Unternehmen wie der KfW, den Stadtwerken München oder dem Deutschen Milchkontor zu tun. An der Spitze ihrer Aufsichtsräte stehen zumeist Arbeiter-, 
Abb. 5 Die soziale Herkunft der Aufsichtsratsvorsitzenden der 100 größten deutschen Unternehmen. (Quelle: eigene Berechnungen)

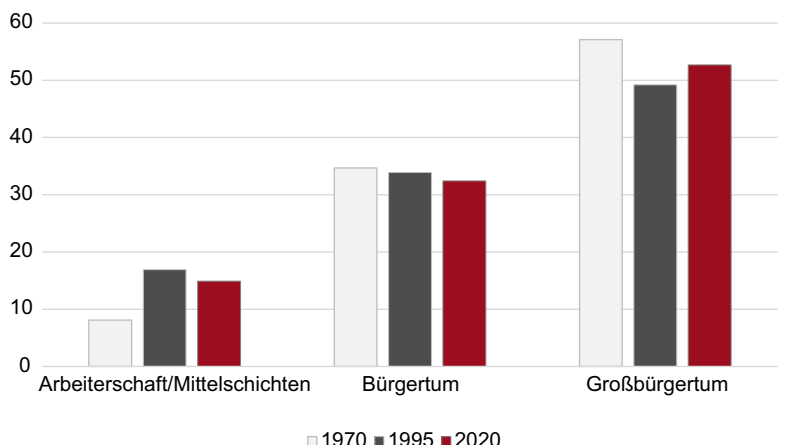

1970 = 1995 =2020

Angestellten- oder Bauernkinder wie in den drei genannten Fällen Bundeswirtschaftsminister Peter Altmaier, Münchens Oberbürgermeister Norbert Reiter oder Heinz Korte. Sie machen ungefähr die Hälfte derjenigen Aufsichtsratsvorsitzenden aus, die aus den Mittelschichten oder der Arbeiterschaft stammen. Hier schlägt sich die im Durchschnitt deutlich breitere soziale Rekrutierung der politischen wie der genossenschaftlichen Spitzenvertreter nieder (Hartmann 2013, S. 52 f.).

\section{Fazit}

Lässt man alle Ergebnisse noch einmal Revue passieren, so lässt sich in Bezug auf die Ausgangsfragen zunächst konstatieren, dass die Lehre, die Hauskarriere und das ingenieur- oder naturwissenschaftliche Studium für soziale Aufsteiger nach wie vor von ganz zentraler Bedeutung sind. Knapp jeder zweite von ihnen hat eine Lehre absolviert, verglichen mit nur gut jedem sechsten bei den Bürger- und Großbürgerkindern. Bei den Hauskarrieren sieht es ähnlich, wenn auch nicht ganz so deutlich aus. Die Aufsteiger haben ihr Berufsleben (bis eventuell auf die ersten vier Jahre) zu über $70 \%$ in einem einzigen Unternehmen verbracht, die anderen nur zu knapp der Hälfte. Schließlich sind Ingenieure und Naturwissenschaftler unter ihnen mit gut $50 \%$ ebenfalls stärker vertreten als im Rest, wo das nicht einmal auf jeden dritten zutrifft. Die Stabilität bei der Bedeutung der Hauskarriere sowie des ingenieur- bzw. naturwissenschaftlichen Studiums kommt sozialen Aufsteigern also entgegen. Der Bedeutungsverlust der Lehre indes verschlechtert ihre Chancen. Er hat jedoch ein deutlich geringeres Gewicht als die beiden erstgenannten Faktoren, zumal eine gewerbliche Lehre entgegen landläufigen Annahmen für Topmanager, auch die sozialen Aufsteiger unter ihnen, immer schon eine absolute Ausnahme dargestellt hat. Insofern ist die große Konstanz in der sozialen Rekrutierung der Wirtschaftselite erst einmal nicht überraschend.

Überraschend ist allerdings, dass der vollkommene Niedergang der Rechtswissenschaften keinerlei spürbaren Einfluss auf die soziale Zusammensetzung der Wirtschaftselite gehabt hat. 1970 und 1995, als Juristen noch fast jeden dritten Vorstandsvorsitzenden stellten, kamen diese nämlich so gut wie ausnahmslos aus bürgerlichen oder großbürgerlichen Familien und prägten damit die soziale Rekrutierung ganz wesentlich. Das juristische Studium entsprach zur Studienzeit der damaligen Vor- 
standschefs noch ganz und gar dem traditionellen Bild einer vorwiegend von den bürgerlich-großbürgerlichen Kreisen gewählten Herrschaftswissenschaft (Hartmann 1990, S. 27 ff.). Doch ganz offensichtlich spielt das konkrete Studienfach keine so große Rolle, wenn es um die soziale Rekrutierung der Topmanager geht, zumindest solange man kein Fach außerhalb des in der Wirtschaft üblichen Kanons wählt. Die familiäre Herkunft ist dafür umso entscheidender. Deshalb war es für den Nachwuchs des Bürger- oder Großbürgertums auch unproblematisch, auf technischnaturwissenschaftliche Fächer oder, was häufiger der Fall war, auf die Wirtschaftswissenschaften umzusatteln, ohne dadurch ihre Karriereaussichten zu verschlechtern oder gar zu gefährden.

Die großen Unterschiede in der Altersstruktur der Vorstandsvorsitzenden je nach sozialer Herkunft werfen allerdings die Frage auf, ob die soziale Rekrutierung in Zukunft vielleicht doch noch exklusiver wird. Während nämlich jeder zweite unter den sozialen Aufsteigern 60 Jahre oder älter ist und nur jeder siebte 50 Jahre oder jünger, sind beide Altersgruppen bei den Kollegen, die aus Bürger- und Großbürgerfamilien stammen, mit 20 zu knapp 19\% fast gleichstark. Die jüngeren Vorstandschefs kommen also sehr viel häufiger aus diesem Milieu. Unter den vergleichsweise alten sind dagegen die Aufsteiger ganz erheblich überrepräsentiert. Es gibt dafür zwei mögliche Erklärungen. Entweder deutet sich hier ein Trend hin zu noch größerer sozialer Selektivität an, oder die Aufsteiger benötigen einfach nur mehr Zeit, bis sie in die Spitzenpositionen gelangen. Welche dieser Erklärungen zutreffend ist oder ob eventuell beide eine Rolle spielen, wird erst das nächste Jahrzehnt zeigen. Die Altersstruktur spricht jedenfalls dagegen, dass es in absehbarer Zukunft zu einer sozialen Öffnung kommen wird. Die Stabilität in der sozialen Zusammensetzung der Wirtschaftselite (Vorstands- und Aufsichtsratsvorsitzende) bedeutet auch, dass es von dieser Seite her keinen Veränderungsimpuls in puncto horizontale Elitenintegration gibt.

Das gilt auch dann noch, wenn man die Nationalität der entsprechenden Personen mit in die Betrachtung einbezieht. Zwar hat die Zahl ausländischer Vorstandschefs zwischen 1995 und 2015 erheblich zugenommen, von gerade einmal zwei auf 15, und unter ihnen sind auch immer häufiger Männer zu finden, die nicht mehr aus sprachlich wie kulturell eng verwandten Ländern stammen - dieser Trend ist aber in den letzten Jahren spürbar gebrochen worden. Aktuell liegt man diesbezüglich wieder auf dem Niveau von 2005, d.h. ein Drittel unter dem des Jahres 2015. ${ }^{20}$ Ähnliche Entwicklungen lassen sich auch in anderen Ländern beobachten, gerade in jenen, in denen die Internationalisierung der Wirtschaftselite in den letzten zwei, drei Jahrzehnten weit vorangeschritten war. Das ist zum einen die Schweiz, die in dieser Beziehung weltweit traditionell den Spitzenplatz einnimmt. Dort ist der Anteil ausländischer CEOs bei den größten Unternehmen seit 2015 ganz massiv von fast drei Vierteln auf nur noch zwei Fünftel gesunken, und von den ausländischen CEOs stellen Deutsche zudem jeden vierten. Von Personen ohne Deutsch als Muttersprache wird aktuell also nicht einmal mehr ein Drittel der Unternehmen geleitet, während es vor fünf Jahren noch fast jedes zweite war (Hartmann 2016,

${ }^{20}$ Die soziale Rekrutierung der ausländischen Vorstandschefs unterschied und unterscheidet sich im Übrigen nicht von der ihrer deutschen Kollegen. 
S. 32). Zum anderen ist es Großbritannien, das vor fünf Jahren von allen großen Industrieländern mit einem Drittel den mit Abstand höchsten Ausländeranteil unter den CEOs der 100 größten Unternehmen aufwies. Dort ist zwar nur ein leichter Rückgang um knapp 10\% zu verzeichnen, das aber nach einer von 1995 bis 2015 sehr schnell und ungebrochen verlaufenen Entwicklung. Selbst in Ländern, die gegenüber ausländischen CEOs traditionell sehr verschlossen waren und sind, zeigt sich ein vergleichbares Bild. So hat der einzige Ausländer, der es an die Spitze eines italienischen Großunternehmens geschafft hat, der Franzose Jean Pierre Mustier bei UniCredit, für Anfang 2021 seinen Rücktritt angekündigt. Als Hauptgrund nannte er tiefgreifende Differenzen mit dem Verwaltungsrat über die zukünftige Strategie. Im Mittelpunkt steht dabei die vom Verwaltungsrat der Bank und von der italienischen Regierung gewünschte Übernahme der wegen eines drohenden Zusammenbruchs verstaatlichten Bank Monte dei Paschi die Siena. Mustier lehnt das entschieden $\mathrm{ab}$, Verwaltungsrat und Regierung wollen es aber unbedingt, weil die EU bei der Zustimmung zur staatlichen Rettung vor vier Jahren die schnelle Reprivatisierung verlangt hat und ihnen die Übernahme als die aus italienischer Sicht beste Variante erscheint.

Der Konflikt bei UniCredit weist auf eine grundlegende Veränderung in den letzten Jahren hin. Die Zunahme nationalistischer Tendenzen in vielen Staaten, vor allem aber bei den traditionellen Vorreitern der Globalisierung, den USA und Großbritannien, lässt die Rolle des Nationalstaates in anderem Licht erscheinen. Er hat auch aus Sicht der meisten Topmanager deutlich an Bedeutung gewonnen. Die Corona-Krise macht das aktuell noch einmal ganz unmissverständlich klar. Vor diesem Hintergrund haben Entwicklungen wie in der Schweiz, wo die umfassende Internationalisierung der Wirtschaftselite deren Verbindungen zur politischen Elite erheblich verschlechtert hat (Mach et al. 2016, S. $121 \mathrm{ff}$.), oder in Großbritannien, wo es der inzwischen ebenfalls stark internationalisierten Finanzelite im Falle des Brexit erstmals seit über 100 Jahren nicht gelungen ist, die Mehrheit der Tories auf ihren Kurs einzuschwören (Hartmann 2017, S. 31 ff.), die Wirtschaftseliten in den meisten Industriestaaten nachdenklich werden lassen. Sie haben gezeigt, wie wichtig intensive Kontakte zur politischen Elite sind und dass eine zu starke Internationalisierung der Wirtschaftselite diese infrage stellen und damit zum Problem werden kann.

Trotz der alles in allem erstaunlich hohen Stabilität, was die „Besonderheiten des Führungspersonals" in den deutschen Großunternehmen angeht, hat die These vom Ende der alten Deutschland AG hinsichtlich der Wirtschaftselite in einem anderen Punkt Recht behalten. Zwar haben sich die Prognosen in Bezug auf das operativ tätige Spitzenmanagement zum größten Teil nicht bestätigt - bei den Aufsichtsratsvorsitzenden sieht das allerdings etwas anders aus. Auch wenn es dort in Bezug auf die soziale Rekrutierung ebenfalls eine hohe Stabilität und Exklusivität gibt, so gehören die lange Jahrzehnte üblichen Mehrfachmandate und die zentrale Rolle der Bankmanager in diesen Gremien tatsächlich der Vergangenheit an. Es gibt derzeit nur noch drei Personen, die jeweils zwei solcher Positionen besetzen, Karl-Ludwig Kley, Wolfgang Reitzle und Nikolaus von Bomhard - keiner von ihnen Repräsentant einer Bank. Vor 25 Jahren wurden noch 21 Aufsichtsräte von Männern geleitet, die mehr als ein derartiges Mandat ausübten. 1970 traf die Mehrfachmandatierung sogar auf 27 Unternehmen zu, mehr als jedes dritte, das damals einen Aufsichtsrat 
besaß. Hermann Josef Abs, der legendäre Vorstandssprecher der Deutschen Bank, war dabei der ungekrönte König mit gleich zehn von diesen Aufsichtsratsvorsitzen, acht davon außerhalb des Finanzsektors. ${ }^{21} 1995$ führten Wolfgang Röller von der Dresdner Bank, Hilmar Kopper von der Deutschen Bank und Friedel Neuber von der Westdeutschen Landesbank zusammen auch noch neun Aufsichtsräte, ebenfalls acht davon außerhalb des Bankensektors. Zählt man andere Bankvorstände wie z.B. Andreas Kleffel, Ronaldo Schmitz oder Franz Heinrich Ullrich, alle drei von der Deutschen Bank, dazu, kommt man 1970 auf 18 aus dem Bankmanagement stammende Aufsichtsratsvorsitzende, die diese Position außerhalb des Finanzsektors bekleideten, und 1995 immerhin noch auf zwölf. Heute trifft das mit Jürgen Fitschen, dem ehemaligen Co-Vorstandschef der Deutschen Bank, nur noch auf einen einzigen Bankmanager zu. Die Kapitalentflechtung zwischen den Großkonzernen der deutschen Wirtschaft und vor allem die zwischen den Banken und den sonstigen Unternehmen hat in dieser Beziehung ganz offensichtlich die erwarteten Konsequenzen gezeitigt.

Dennoch täuscht auch dieser Eindruck zumindest ein wenig; denn immerhin noch fast zwei Fünftel der aktuellen Aufsichtsratsvorsitzenden saßen zuvor im Vorstand eines dieser Großunternehmen, teilweise desselben, zu gut zwei Dritteln aber, wie Reitzle oder Kley, in dem eines anderen. ${ }^{22}$ Verflechtungen zwischen den Großunternehmen gibt es also weiterhin, wenn auch längst nicht mehr so ausgeprägt wie in den Zeiten der alten Deutschland AG und im Unterschied zu früher weitgehend ohne Beteiligung der Bankmanager. Dafür spricht auch die Tatsache, dass die Anzahl der Politiker oder hohen politischen Beamten an der Spitze der Aufsichtsgremien über den gesamten Zeitraum ausgesprochen stabil geblieben ist, mit gut einer Handvoll allerdings auf einem relativ niedrigem Niveau. Beyers Feststellung, dass Unternehmen mit einem Wirtschaftswissenschaftler an der Spitze personell weniger verflochten sind, hat sich bei den Aufsichtsratsvorsitzenden im Übrigen nicht bestätigt. Es gibt bei den Unternehmen, deren Aufsichtsrat von einem externen Manager geleitet wird, so gut wie keine Unterschiede, was die Studienfächer der Vorstandschefs angeht.

Mit Blick auf die anfangs zitierte Aussage von Schluchter muss man abschließend also ein eher nüchternes Fazit ziehen, vor allem wenn man sie vor dem Hintergrund der Prognosen von Streeck und anderen betrachtet. Trotz großer politischer wie wirtschaftlicher Umwälzungen hat sich die Rekrutierung der Wirtschaftselite in den letzten 50 Jahren als außergewöhnlich stabil erwiesen. Der in den 1990er-Jahren prophezeite Siegeszug des MBA ist ebenso ausgeblieben wie der damals lautstark angekündigte Vormarsch der Privathochschulen. Die einzige wirklich tiefgreifende, von den allermeisten Beobachtern allerdings nicht erwartete Veränderung besteht im dramatischen Niedergang der Juristen. Sie sind binnen gut zwei Jahrzehnten von der mächtigsten Akademikergruppe in den Chefetagen der deutschen Wirtschaft zu einem Randphänomen geworden. Dass ein Studienabschluss mittlerweile zur

\footnotetext{
21 Vor der Novellierung des Aktiengesetzes 1965 waren es sogar 20. Dieses auch als „Lex Abs“ bekannt gewordene Gesetz sollte seine Machtfülle durch eine maximale Anzahl von zehn Aufsichtsratsvorsitzen für eine Person verringern.

${ }^{22}$ Der zukünftige Aufsichtsratsvorsitzende von Daimler, der frühere BMW- und VW-Chef Bernd Pischetsrieder, passt in dieses Bild.
} 
„Conditio sine qua non“ geworden ist und die Lehre an Bedeutung eingebüßt hat, verwundert angesichts der Bildungsexpansion der letzten Jahrzehnte dagegen nicht. $\mathrm{Zu}$ der aufgrund dieser Bildungsexpansion vielfach erhofften sozialen Öffnung der Wirtschaftselite ist es allerdings nicht gekommen. Ein Anstieg um gerade einmal zwei Prozentpunkte, was den Anteil der sozialen Aufsteiger an den Vorstandsvorsitzenden angeht, ist für ein halbes Jahrhundert so gut wie nichts. Dieser Eindruck ändert sich auch nicht, wenn man die etwas niedrigeren Werte für 1995 und 2005 zum Vergleich heranzieht. Es bleibt dabei, dass konstant mehr als vier Fünftel der Vorstandschefs aus bürger- oder großbürgerlichen Familien stammen und Arbeiterkinder trotz aller prominenten Beispiele (wie z.B. Herbert Diess bei VW) durchweg eine Rarität darstellen.

Für die horizontale Elitenintegration bedeutet das in Hinblick auf den zentralen Faktor soziale Homogenität ebenfalls Stabilität. Es ist von Seiten der Wirtschaftselite sozialstrukturell weder zu einer Vertiefung der Unterschiede zu den anderen Teileliten gekommen noch zu einer Annäherung. Es gibt nur Veränderungen in einzelnen, nicht wirklich gravierenden Punkten. So haben die direkten familiären Beziehungen zur politischen Elite (im weiteren Sinne) zugenommen. Hatte von den Vorstandsvorsitzenden der Jahres 1970 und 1995 niemand einen Politiker zum Vater, geschweige denn einen einflussreichen, sondern allenfalls einen hohen Richter (bei Helmut Horten und Hans Samwer 1970 jeweils Senatspräsidenten) oder einen hohen Verwaltungsbeamten (bei Ulrich Hartmann, Dietmar Kuhnt und Jörg von Craushaar 1995 ein Staatsekretär, ein Oberstadtdirektor und ein Regierungspräsident), so gilt das aktuell immerhin schon für zwei Vorstandschefs. Christian Kleins (SAP) Vater ist aktuell Vorsitzender des Innenausschusses im baden-württembergischen Landtag und Simon Nüssels (Baywa) Vater war bayerischer Staatsminister für Ernährung, Landwirtschaft und Forsten. Gleichzeitig hat sich der Anteil der höheren Justiz- und Verwaltungsjuristen unter den Vätern im gesamten Zeitraum kaum verändert. Er bewegt sich ohne nennenswerte Schwankungen um die zehn Prozent herum. Stabilität in puncto soziale Herkunft charakterisiert auch die beiden nach der Wirtschaftselite sozial exklusivsten Eliten, die aus Justiz und Verwaltung, mit ihren ungefähr zwei Dritteln an Bürger- oder Großbürgerkindern. Da die vierte zentrale Elite, die aus der Politik, in den letzten zwei Jahrzehnten aber sozial an Exklusivität gewonnen hat, kann man insgesamt sogar von einer moderaten Stärkung der Elitenintegration sprechen, soweit es ihre soziale Herkunft betrifft (Hartmann 2013, S. 64 ff.).

Selbst der deutliche Anstieg ausländischer Vorstandschefs zwischen 1995 und 2015 dürfte diesen Effekt nicht groß konterkariert haben: Sie wurden nämlich in den meisten Fällen nicht extern rekrutiert, sondern waren schon mindestens ein Jahrzehnt bei dem Unternehmen tätig, dessen Leitung sie letztlich übernahmen. AuBerdem hatten sie vor ihrer Ernennung auch schon etliche Jahre in Deutschland gelebt. Dieses Muster hat sich seither fortgesetzt, allerdings auf (rein quantitativ) deutlich reduziertem Level. So hat z.B. der neue Vorstandsvorsitzende von Daimler, der Schwede Ola Källenius, seine gesamte Karriere in dem Unternehmen absolviert und nach eineinhalb Jahrzehnten Tätigkeit in den USA und Großbritannien die letzten zehn Jahre bereits in Deutschland gearbeitet und gelebt. Es gab und gibt nur wenige, die wie John Cryan relativ kurzfristig aus dem Ausland in ein Unternehmen 
geholt worden sind..$^{23}$ Nur sie dürften keine engere Bindung zu den anderen Eliten in Deutschland gefunden haben. Alles in allem gibt es in Hinblick auf die wesentlichen Merkmale, vor allem die soziale Rekrutierung der Wirtschaftselite, vorwiegend Kontinuität und nur vergleichsweise selten durchgreifenden Wandel.

Eine mögliche Erklärung dafür könnte in den Eigentümer- und damit auch Machtstrukturen innerhalb der deutschen Wirtschaft zu finden sein. Ein im internationalen Vergleich außergewöhnlich hoher Anteil der Großunternehmen befindet sich nämlich durchgängig unter Familienkontrolle. Schon 1970 traf das auf knapp ein Drittel, 1995 auf deutlich mehr als ein Drittel und heute auf fast die Hälfte zu. Diese Struktur sorgt für Kontinuität in den Unternehmen und wohl auch in den Rekrutierungskriterien. Sie konterkariert gerade durch ihren nochmaligen Bedeutungszuwachs seit 1995 den zeitgleich stattfindenden Niedergang der klassischen Deutschland AG. Auch wenn in den Medien der Einfluss ausländischer Anteilseigner auf die DAXKonzerne immer wieder thematisiert wird, allen voran der von Blackrock, darf man nicht übersehen, dass selbst unter diesen 30 Unternehmen über ein Viertel von Eigentümerfamilien kontrolliert wird - wie z.B. VW, BMW, Henkel oder Merck. Die von Streeck und anderen um die Jahrtausendwende prognostizierte Angleichung des deutschen Managementtypus an den angelsächsischen könnte hier ihre Grenzen gefunden haben.

Funding Open Access funding enabled and organized by Projekt DEAL.

Open Access Dieser Artikel wird unter der Creative Commons Namensnennung 4.0 International Lizenz veröffentlicht, welche die Nutzung, Vervielfältigung, Bearbeitung, Verbreitung und Wiedergabe in jeglichem Medium und Format erlaubt, sofern Sie den/die ursprünglichen Autor(en) und die Quelle ordnungsgemäß nennen, einen Link zur Creative Commons Lizenz beifügen und angeben, ob Änderungen vorgenommen wurden.

Die in diesem Artikel enthaltenen Bilder und sonstiges Drittmaterial unterliegen ebenfalls der genannten Creative Commons Lizenz, sofern sich aus der Abbildungslegende nichts anderes ergibt. Sofern das betreffende Material nicht unter der genannten Creative Commons Lizenz steht und die betreffende Handlung nicht nach gesetzlichen Vorschriften erlaubt ist, ist für die oben aufgeführten Weiterverwendungen des Materials die Einwilligung des jeweiligen Rechteinhabers einzuholen.

Weitere Details zur Lizenz entnehmen Sie bitte der Lizenzinformation auf http://creativecommons.org/ licenses/by/4.0/deed.de.

\section{Literatur}

Beyer, J. (1998). Managerherrschaft in Deutschland? „Corporate Governance“ unter Verflechtungsbedingungen. Opladen: Westdeutscher Verlag.

Beyer, J. (1999). Unternehmensverflechtungen und Managerherrschaft in Deutschland. Leviathan, 27, $518-536$.

Beyer, J. (2006). Vom Netzwerk zum Markt? Zur Kontrolle der Managementelite in Deutschland. In W. Streeck \& M. Höpner (Hrsg.), Alle Macht dem Markt? Fallstudien zur Abwicklung der Deutschland $A G$ (S. 177-198). Frankfurt a. M.: Campus.

23 Aktuell trifft das nur auf Mark Garrett, den Vorstandsvorsitzenden von Marquardt\&Bahls, zu. Er ist erst 2018 nach Deutschland gekommen, war zuvor allerdings über zehn Jahre in derselben Position beim österreichischen Konzern Borealis, lebte also bereits seit langen Jahren in einer deutschsprachigen Umgebung. 
Byrkjeflot, H. (2000). Management education and selection of top managers in Europe and the United States. LOS-Center Rapport 0103. Bergen.

Gergs, H.-J., \& Schmidt, R. (2002). Generationswechsel im Management ost- und westdeutscher Unternehmen. Kommt es zu einer Amerikanisierung des deutschen Managementmodells? Kölner Zeitschrift für Soziologie und Sozialpsychologie, 54, 553-578.

Hartmann, M. (1990). Juristen in der Wirtschaft. Eine Elite im Wandel. München: C.H. Beck.

Hartmann, M. (1996). Topmanager. Die Rekrutierung einer Elite. Frankfurt a. M.: Campus

Hartmann, M. (1997). Soziale Öffnung oder soziale Schließung. Die deutsche Wirtschaftselite zwischen 1970 und 1995. Zeitschrift für Soziologie, 26, 296-311.

Hartmann, M. (1999). Auf dem Weg zu einer transnationalen Bourgeoisie? Leviathan, 27, 113-141.

Hartmann, M. (2002). Der Mythos von den Leistungseliten. Spitzenkarrieren und soziale Herkunft in Wirtschaft, Politik, Justiz und Wissenschaft. Frankfurt a. M.: Campus.

Hartmann, M. (2007). Eliten und Macht in Europa. Frankfurt a. M.: Campus.

Hartmann, M. (2009). Die transnationale Klasse - Mythos oder Realität? Soziale Welt, 60, 285-303.

Hartmann, M. (2013). Soziale Ungleichheit. Kein Thema für die Eliten? Frankfurt a. M.: Campus.

Hartmann, M. (2016). Die globale Wirtschaftselite. Eine Legende. Frankfurt a. M.: Campus.

Hartmann, M. (2017). Brexit - on the declining homogeneity of European elites - and on the importance of a domestic habitus in times of globalization. Culture, Practice and European Policy, 2, $28-34$.

Hartmann, M. (2018). Die Abgehobenen. Wie die Eliten die Demokratie gefährden. Frankfurt a. M.: Campus.

Hassel, A., Höpner, M., Kurdelbusch, A., Rehder, B., \& Zugehör, R. (2000). Zwei Dimensionen der Internationalisierung: Eine empirische Analyse deutscher Großunternehmen. Kölner Zeitschrift für Soziologie und Sozialpsychologie, 52, 500-519.

Höpner, M. (2003). Wer beherrscht die Unternehmen? Shareholder Value, Managerherrschaft und Mitbestimmung in Deutschland. Frankfurt a. M.: Campus.

Höpner, M. (2004). Was bewegt die Führungskräfte? Von der Agency-Theorie zur Soziologie des Managements. Soziale Welt, 55, 263-282.

Höpner, M., \& Jackson, G. (2001). Entsteht ein Markt für Unternehmenskontrolle? Der Fall Mannesmann. Leviathan, 29, 544-563.

Höpner, M., \& Jackson, G. (2002). Das deutsche System der Corporate Governance zwischen Persistenz und Konvergenz. Kölner Zeitschrift für Soziologie und Sozialpsychologie, 54, 362-368.

Lane, C. (1989). Management and labour in Europe. The industrial enterprise in Germany, Britain and France. Aldershot: Edward Elgar.

Locke, R. (1989). Management and higher education. Cambridge: Cambridge University Press.

Mach, A., David, T., Ginalski, S., \& Bühlmann, F. (2016). Les élites économiques suisses au XXe siècle. Neuchâtel: Éditions Alphil.

Petzina, D., Abelshauser, W., \& Faust, A. (1978). Sozialgeschichtliches Arbeitsbuch III. Materialien zur Geschichte des Deutschen Reiches 1914-1945. München: C.H. Beck.

Schluchter, W. (1963). Der Elitebegriff als soziologische Kategorie. Kölner Zeitschrift für Soziologie und Sozialpsychologie, 15, 233-256.

Sorge, A. (1999). Mitbestimmung, Arbeitsorganisation und Technikanwendung. In W. Streeck \& N. Kluge (Hrsg.), Mitbestimmung in Deutschland. Tradition und Effizienz (S. 17-134). Frankfurt a. M.: Campus.

Stewart, R., Barsoux, J.-L., Kieser, A., Ganter, H.-D., \& Walgenbach, P. (1994). Managing in Britain and Germany. London: Anglo-German Foundation for the Study of Industrial Society.

Streeck, W. (2006). Nach dem Korporatismus: Neue Eliten, neue Konflikte. In W. Streeck \& M. Höpner (Hrsg.), Alle Macht dem Markt? Fallstudien zur Abwicklung der Deutschland AG (S. 149-176). Frankfurt a. M.: Campus.

Streeck, W., \& Höpner, M. (2003). Einleitung: Alle Macht dem Markt. In W. Streeck \& M. Höpner (Hrsg.), Alle Macht dem Markt? Fallstudien zur Abwicklung der Deutschland AG (S. 11-59). Frankfurt a. M.: Campus.

Michael Hartmann geb. 1952. Prof. Dr. i.R., Technische Universität Darmstadt. Forschungsschwerpunkte: Eliten- und Managementforschung im internationalen Vergleich. Ausgewählte Veröffentlichungen: Der Mythos von den Leistungseliten, 2002; Soziale Ungleichheit - Kein Thema für die Eliten?, 2013; Die globale Wirtschaftselite. Eine Legende, 2016; Die Abgehobenen. Wie die Eliten die Demokratie gefährden, 2018 